\title{
Neuroleptika im Einsatz
}

\section{Sehr geehrte Kolleginnen und Kollegen,}

Sie halten heute gewissermaßen ein „Doppelheft“ der Zeitschrift psychoneuro in Händen, das sich diesmal sowohl an Nervenärzte, Neurologen und Psychiater als auch an Hausärzte richtet. Das Schwerpunktthema „Neuroleptika im Einsatz“ wurde daher mit drei Übersichtsarbeiten zu psychischen Erkrankungen in der Praxis ergänzt. Grundlage für die Überlegungen zur Herausgabe eines gemeinsamen Heftes an die genannten Berufsgruppen ist die Jahrestagung der Deutschen Gesellschaft für Psychiatrie, Psychotherapie und Nervenheilkunde (DGPPN) vom 19.-22.11.2003 in Berlin, die diesmal zusammen mit der Fachgesellschaft und dem Berufsverband der Fachärzte für Allgemeinmedizin stattfindet.

Hausärzte (Allgemeinmediziner, Internisten, Praktische Ärzte) und Nervenärzte/Psychiater sind auf eine enge Zusammenarbeit angewiesen. Zahlreiche Untersuchungen belegen, dass mindestens $20 \%$ der Patienten in einer hausärztlichen Praxis an einer psychischen Störung leiden $(1,3)$. Auf der anderen Seite zeigt die Erfahrung, dass psychiatrische Patienten in fachärztlicher Behandlung in ca. $40 \%$ behandlungsbedürftige körperliche Erkrankungen aufweisen, die vom Hausarzt versorgt werden müssen (2). Fachübergreifende Diagnostik und Behandlung sind in der Regel notwendig z.B. bei psychiatrischen Erkrankungsbildern im Rahmen endokriner Störungen, systemischer oder isolierter zentralnervöser Entzündungen und Tumorerkrankungen bzw. in der Folge einer medikamentösen Therapie somatischer Erkrankungen (z.B. durch Antihypertensiva, Antibiotika, Analgetika, Anticholinergika). Darüber hinaus stellen typische psychiatrische Erkrankungen wie z.B. eine Depression mit somatischem Syndrom hohe Anforderungen an die gemeinsame Behandlung der Patienten. Aber auch die hausärztliche Weiterbehandlung der Patienten, die von Nervenärzten oder Psychiatern primär diagnostiziert und therapiert wurden, ist an dieser Stelle zu nennen. Eine Übersicht über diese Störungen, häufige Anfragen durch den Hausarzt an den Facharzt und die Probleme in der Zusammenarbeit gibt der Beitrag von Deckert in diesem Heft.

Der Wissenszuwachs in der Psychiatrie und in der Allgemeinmedizin war in den letzten zehn Jahren rasant. Neue Zusammenhänge zwischen körperlichen und psychischen Störungen wurden entdeckt bzw. gesichert - z.B. ein Zusammenhang zwischen Höhe des Cholesterinspiegels und der Inzidenz von Angsterkrankungen und Depressionen (4) -, neurobiologische Korrelate unterschiedlicher psychischer Erkrankungen wurden beschrieben, diagnostische Kriterien für psychiatrische Störungen durch DSM-IV und ICD-10 weiterentwickelt und damit die Diagnostik weiter differenziert. Hinsichtlich der Therapie sind eine Fülle neuer Medikamente mit spezifischen Indikationsbereichen und neuen pharmakologischen Wirkweisen (und Interaktionen) auf den Markt gekommen. Bereits aus fachärztlich psychiatrischer Sicht ist es schwer, hierüber den Überblick zu bewahren. Für Hausärzte, die sich in vielen weiteren Disziplinen weiterbilden müssen, ist das oft unmöglich. In diesem Heft sollen einige Störungsbilder angesprochen werden, deren Erkennung und Behandlung aus psychiatrischer Sicht für Haus- und
Fachärzte gleichermaßen wichtig ist. Der Beitrag von Grabe und Freyberger beschäftigt sich mit den Patienten, die sich mit unspezifischem Beschwerdebild und ohne wegweisende organmedizinische Befunde immer wieder beim Hausarzt vorstellen, und gibt eine Übersicht über die Diagnostik und Therapie der Somatisierungsstörung. Hambrecht beschreibt die Frühsymptomatik von Psychosen und verweist darauf, dass die Früherkennung und Behandlung von hoher Relevanz für die Prognose dieser Erkrankungen ist. Holzbach bewertet neue Antipsychotika hinsichtlich ihrer Effektivität für die Akuttherapie und betont die Bedeutung einer patientenangemessenen Therapie, damit die Einnahme der Medikamente gewährleistet ist. Retz et al. stellen die Bedeutung der Diagnosestellung und Behandlung von Aufmerksamkeits- und Hyperaktivitätsstörungen (ADHS) im Erwachsenenalter dar.

Allen genannten Störungsbildern ist gemeinsam, dass die betroffenen Patienten oft primär vom Hausarzt gesehen werden und später interdisziplinär von Haus- und Facharzt weiter betreut werden (5). Das vorliegende Heft möchte deshalb die gemeinsame Verantwortung beider Berufsgruppen für eine angemessene Kooperation sowohl hinsichtlich der Diagnostik und Therapie als auch in der Fortbildung hervorheben. Ich hoffe in diesem Sinne, dass das Heft eine anregende und lehrreiche Auswahl der Themen getroffen hat.

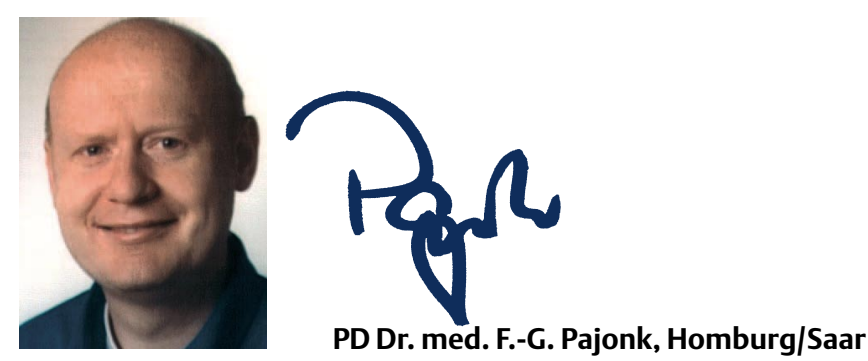

\section{Literatur}

1. Jacobi F, Höfler M, Meister W, Wittchen HU. Prävalenz, Erkennens- und Verschreibensverhalten bei depressiven Syndromen. Eine bundesdeutsche Hausarztstudie. Nervenarzt 2002; 73: 651-658

2. Jacobi F, Wittchen HU. Comorbidity of mental and somatic disorders in the general population. Posterpräsentation auf der Jahrestagung der Arbeitsgemeinschaft Neuropsychopharmakologie (AGNP), München, 9.-11.10.2003

3. Linden M, Maier W, Achberger M, Herr R, Helmchen H, Benkert O. Psychiatrische Erkrankungen und ihre Behandlung in Allgemeinarztpraxen in Deutschland: Ergebnisse einer Studie der WHO. Nervenarzt 1996; 67: 205-215

4. Sevincok L, Buyukozturk A, Dereboy F. Serum lipid concentrations in patients with comorbid generalized anxiety disorder and major depressive disorder. Can J Psychiatry 2001; 46: 68-71

5. Wittchen HU, Jacobi F. Die Versorgungssituation psychischer Störungen in Deutschland. Bundesgesundheitsblatt - Gesundheitsforschung - Gesundheitsschutz 2001; 44 